\title{
Enterostomal Therapist
}

National Cancer Institute

\section{Source}

National Cancer Institute. Enterostomal Therapist. NCI Thesaurus. Code C19180.

A healthcare professional trained to help patients adjust to and care for their colostomy. 